\title{
ECG signal Analysis and Classification Techniques
}

\author{
Seema Punia, Dinesh Kumar Atal, Sarita Singh
}

\begin{abstract}
Electrocardiogram is the measure of heart electrical activity. Our heart generate electrical signals which we used to calculate heart activity. The electrical signals of heart are transformed into waveforms which are used to measure various heart conditions. We have various techniques which we used to analyze and classified the ECG signals in MATLAB. There are many types of heart Arrhythmia like Tachycardia in which heart rate is too fast, Bradycardia in which heart rate is too slow, Atrial Fibrillation, Atrial Flutter, Ventricular Fibrillation,Permature contractions these all conditions can easily classified in Matlab by using some proper approach. We have techniques like Wavelet transform, Graphical user interface using wavelet transform toolbox, Support vector machine, Convolutional neural network, Discrete cosine transform. To improve the order execution, molecule swarm improvement method is utilized for progressively tuning the learning parameters of the SVM classifier. This paper gives brief survey on different techniques for analysis and classification of ECG signals. Wavelet Transform gives more accuracy and precise result. And we analyze MATLAB software is a best approach for analysis and classification of ECG signals. Keywords : ECG; MATLAB; Electrical Signal; Wavelet transform; Convolutional neural network; Support vector machine.
\end{abstract}

\section{INTRODUCTION}

Electrocardiogram is estimation of heart movement. Our heart is comprised of four sections two sections are atria and two are ventricles upper part both left and right atria and lower part both left and right ventricles [1]. Heart create electrical signs the age of electrical signs happens by the sinoatrial hub or sinus hub .SA hub is called characteristic pacemaker of the heart.ECG is comprised of five waves $\mathrm{P}, \mathrm{Q}, \mathrm{R}, \mathrm{S}$ and $\mathrm{T}$ wave. The electrical signs which are created by Sinoatrial hub [3] ascertain by setting the anodes on the outside of the body and these signs are produced as waveform [17]. These waveforms are called P wave, Q wave, $\mathrm{R}$ wave, $\mathrm{S}$ wave, and $\mathrm{T}$ wave waveforms. $\mathrm{P}$ waveform is happened when withdrawal of the privilege and left atria happened. QRS complex happened when the privilege and left ventricular depolarization happens. The $\mathrm{T}$ waveform is happen when the ventricular unwinding happens and $U$ waveform presumably after the constriction in the ventricles.

Revised Manuscript Received on April, 042020.

* Correspondence Author

Seema Punia*, Department of Biomedical Engineering, Deenbandhu Chhotu Ram University of Science and Technology, Murthal-131039, Sonipat, Haryana, India

Dinesh Kumar Atal, Department of Biomedical Engineering, Deenbandhu Chhotu Ram University of Science and Technology, Murthal131039, Sonipat, Haryana, India

Sarita Singh, Department of Biomedical Engineering, Deenbandhu Chhotu Ram University of Science and Technology, Murthal-131039, Sonipat, Haryana, India

(C) The Authors. Published by Blue Eyes Intelligence Engineering and Sciences Publication (BEIESP). This is an open access article under the CC BY-NC-ND license (http://creativecommons.org/licenses/by-nc-nd/4.0/)
By recognizing proposals waveforms we can investigate and arranged the ECG signals.QRS complex and R-top show the warmth rate inconstancy and different conditions [13]. There are some standard estimations of ECG various parameters. For R-R interim, interim length is $0.6-1.20$ second ,for T-T interim, interim term is $0.6-1.20$ second, for ST portion 0.08-0.12 second, PR interim 0.12-0.20 seconds, QT interim 0.35-0.44 second, PP interim 0.6-1.20 second, QRS interim 0.08-0.12 second, PQ interim 0.12-0.20 second, $\mathrm{P}$ waveform sufficiency is $0.25 \mathrm{mv}$, $\mathrm{R}$ waveform is $1.6 \mathrm{mv}, \mathrm{Q}$ wave adequacy is $25 \%$ vof $\mathrm{R}$ waveform and $\mathrm{T}$ waveform abundance is $0.1-0.5 \mathrm{mv}$ [9]. When theses waveforms are not in suitable form or the electrical signal generated by SA node not working properly then many types of abnormalities occurred in heart which we can classify using various techniques. Aside from the robotized investigation of ECG being critical in clinical cardiology, the human understanding and observing can't satisfy the prerequisites for constant analysis of arrhythmias [22]

\section{METHODS AND MATERIAL}

\subsection{DISCRETE WAVELET TRANSFORM (DWT)}

DWT is which convert or transform the discrete time signals into wavelet representation. In medical field medical images needs more concern without any loss of information. Discrete wavelet transform is a great method which based upon time scale representation and provides good information. In Discrete Wavelet Transform Daubechies wavelet algorithm [3] is more efficient as compared to some other algorithms like Haar algorithm. Discrete wavelet transform is advance version of Fourier transform [9].Fourier transform provide frequency information only whereas discrete wavelet transform provide frequency information and the information about the event in time. In Daubechies wavelets [12] fast implementation and finite number of filter parameters are present. So, basically discrete wavelet transform is an effective tool use to remove signals within specific subband. There are several mother wavelets which are used to convert the signals into time frequency these are Haar, DB, Coieflet, Symlet, and Mexican Hat 30].

\subsection{DIGITAL FILTER APPROACH}

In Matlab digital filters are also use for the removal of noise or interference. With the help of filters we can easily pass a certain frequency components and rejection of others. There are many types of digital filters such as IIR filter which is a filter with an infinite impulse response, FIR filer a filter with finite response both the filters have different properties if we need linear phase then FIR filters have linear phase whereas if we not need linear phase then IIR filter is more efficient to implement then FIR filter [8].

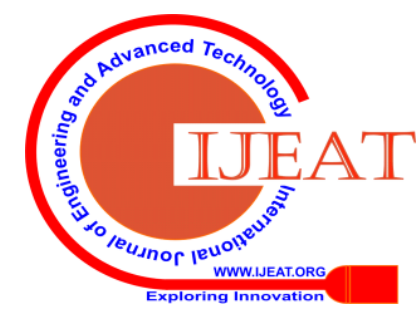


There are some ideal filters which are similarly used for noise and interference removal such filters are High pass channel, low pass channel, Band-stop channel, step channel, Band-pass channel, Baseline wander removing[3].

\subsection{SUPPORT VECTOR MACHINE}

In AI, support-vector machines are managed learning models with related learning calculations that break down information utilized for characterization and relapse investigation. Given a lot of preparing models, each set apart as having a place with either of two classifications, a SVM [31] preparing calculation constructs a model that allots new guides to one classification or the other, making it a nonprobabilistic double straight classifier (in spite of the fact that strategies, for example, Platt scaling exist to utilize SVM in a probabilistic order setting)[29]. A SVM model is a portrayal of the models as focuses in space, mapped with the goal that the instances of the different classes are isolated by a reasonable hole that is as wide as would be prudent. New models are then mapped into that equivalent space and anticipated to have a place with a classification

dependent on the side of the hole on which they fall. A Support vector machine develops a hyperplane or set of hyperplanes in a high-or endless dimensional space 18], which can be utilized for order, relapse, or different assignments like exceptions detection. Intuitively, a great division is accomplished by the hyperplane that has the biggest separation to the closest preparing information purpose of any class, since when all is said in done the bigger the edge, the lower the speculation mistake of the classifier[25][17].

\subsection{FEATURE SELECTION}

The principle motivation behind the element determination is deciding the component subset which gives the most noteworthy segregation between the gatherings. Utilizing all highlights in a classifier doesn't give the best execution as a rule. Highlight choice additionally encourages individuals to secure a superior comprehension about which highlights are significant in diagnosing the information of intrigue.

\subsection{PROJECT FLOW}

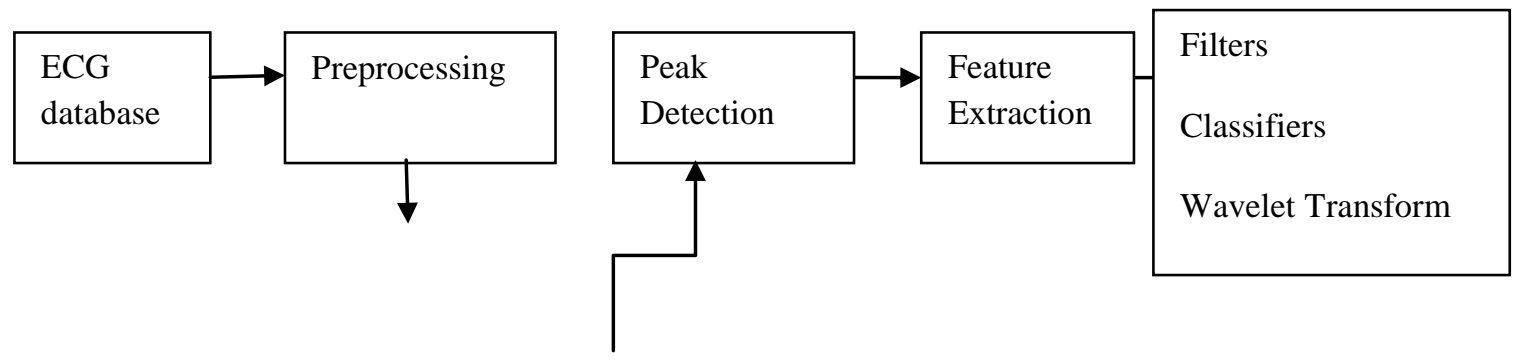

Fig. 1 flow diagram of ECG signal analysis

\subsection{CLASSIFICATION}

In study, Probabilistic Neural Network (PNN)[30], k-means clustering[1], Support Vector machine (SVM)[17], Extreme learning machine and Multi-Layer Perceptron (MLP) were used for the pattern classification.

\section{RESULT AND DISCUSSION}

This paper audits the approach for examining the diverse ECG informational collections. Different obstructions that come in the ECG signal investigation is its nonlinearity, fluctuation, long length and non stationary. The fundamental motivation to give an outline of ECG signal investigation in this paper is to improve the issue limit with the goal that total ECG nonlinearity might be examined successfully. It will help in appropriately grouping arrhythmias. Under uproarious condition only frequency domain methods and time domain methods are not reasonable on the grounds area suitable strategies and recurrence space techniques are most certainly that the areas are not obviously seen utilizing unaided eye of the person just even it requires nonlinear strategies.

Examinations with WT require determination of astounding wavelet premise work for breaking down ECG signals. No system is accessible for choosing wavelet premise work, however it disintegrates the ECG signal into time frequency levels successfully in light of the fact that its activity is represented by scaling and interpretation work, while Fourier isn't fitting for the preparing of the nonstationary elements of the ECG signal. A back propagation calculation with feed forward neural system is utilized for classification that is productive. In any case, the wavelet approach is more helpful than the customary sifting systems, which features the subtleties of the ECG signal with ideal time-recurrence goals. On the off chance that we dissect the ECG signal by both regular FFT and Wavelet change, at that point we have seen Wavelet is as better than the regular FFT technique in finding the little variations from the norm in ECG signals. However, the non-adaptive filter is quicker than the adaptive filter strategies. Since wavelets are utilized for pre-preparing of ECG signal it is discovered that acceptable SNR proportion is acquired utilizing bio-orthogonal wavelet. MATLAB is a compelling device for ECG information examination and variation from the norm recognition. The entire framework is exceptionally valuable for observing the ECG sign and self finding of the heart. LIBSVM doesn't require a lot of tests and is superior to conventional strategies in choosing ideal highlights in the multi-area space vector. Along these lines, this new procedure can be utilized proficiently for ECG analysis.

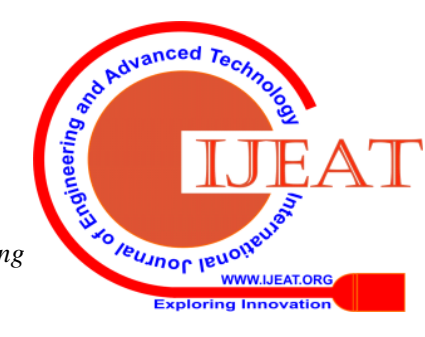


Table 1: Review of Various Papers on analysis \&classification of ECG signals

\begin{tabular}{|c|c|c|c|}
\hline $\begin{array}{l}\text { Authors } \\
\text { \& Year }\end{array}$ & Paper Title & $\begin{array}{l}\text { Technique } \\
\text { used }\end{array}$ & Result \\
\hline $\begin{array}{c}\text { Sung-Nien } \\
\text { Yu } \\
\text { et al. } \\
\text { [30] }\end{array}$ & $\begin{array}{l}\text { ECG beat } \\
\text { classification based } \\
\text { on PNN and } \\
\text { wavelet } \\
\text { transformation }\end{array}$ & $\begin{array}{l}\text { Wavelet } \\
\text { transformatio } \\
\mathrm{n} \text { technique, } \\
\text { Using } \\
\text { Wavelet } \\
\text { technique } \\
\text { extract } \\
\text { features } \\
\text { Probabilistic } \\
\text { Neural } \\
\text { Network(PNN } \\
\text { ) }\end{array}$ & $\begin{array}{l}\text { Three classes of } \\
\text { highlights } \\
\text { comparing to the } \\
\text { vitality, the } \\
\text { cognizance, and } \\
\text { the morphological } \\
\text { attributes were } \\
\text { first extricated } \\
\text { from } \\
\text { theDeteriorated } \\
\text { waveforms. A } \\
\text { PNN classifier } \\
\text { was at that point } \\
\text { utilized to } \\
\text { segregate six } \\
\text { kinds of ECG } \\
\text { beats. The results } \\
\text { show a promising } \\
\text { exactness of } \\
\text { 99.65\%. }\end{array}$ \\
\hline $\begin{array}{l}\text { C.Saritha } \\
\text { et al. } \\
\text { [9] }\end{array}$ & $\begin{array}{l}\text { Analysis of } \\
\text { Electrocardiogram } \\
\text { using WT }\end{array}$ & $\begin{array}{l}\text { Wavelet } \\
\text { transform } \\
\text { analysis } \\
\text { technique } \\
\text { QRS complex } \\
\text { detection } \\
\text { Peak } \\
\text { detection of } \\
\text { each wave } \\
\text { Matlab } \\
\text { software }\end{array}$ & $\begin{array}{l}\text { Accuracy-99.8\% } \\
\text { Calculate various } \\
\text { abnormalities and } \\
\text { their features } \\
\text { compare them } \\
\text { with normal ECG } \\
\text { waveforms. }\end{array}$ \\
\hline $\begin{array}{l}\text { S.karpagach } \\
\text { elvi et al. } \\
\text { [31] }\end{array}$ & $\begin{array}{l}\text { Classification of } \\
\text { ECG signals with } \\
\text { SVM\& ELM }\end{array}$ & $\begin{array}{l}\text { Extreme LM } \\
\text { classifier } \\
\text { MIT-BIH } \\
\text { database }\end{array}$ & $\begin{array}{l}\text { Accuracy-89.78\% } \\
\text { ELM achieves } \\
\text { better and } \\
\text { increasingly } \\
\text { adjusted } \\
\text { characterization } \\
\text { for singular } \\
\text { classifications too } \\
\text { in exceptionally } \\
\text { less preparing } \\
\text { time relative to } \\
\text { SVM }\end{array}$ \\
\hline $\begin{array}{l}\text { Narayana } \\
\text { K.V.L. } \\
\text { et al. } \\
\text { [13] }\end{array}$ & $\begin{array}{l}\text { QRS detection in } \\
\text { Electrocardiogram } \\
\text { based on wavelet } \\
\text { using Matlab }\end{array}$ & $\begin{array}{l}\text { Matlab } \\
\text { software } \\
\text { Wavelet } \\
\text { based } \\
\text { algorithms } \\
\text { Detection of } \\
\text { QRS complex }\end{array}$ & $\begin{array}{l}\text { In comparison of } \\
\text { Pan Tompkins } \\
\text { AF2 algorithm is } \\
\text { fast in detection of } \\
\text { QRS complex } \\
\text { AF2 memory } \\
\text { requirement in } \\
\text { comparison of Pan } \\
\text { Tompkins is less }\end{array}$ \\
\hline $\begin{array}{l}\text { k.Islam M. } \\
\text { et al. } \\
\text { [3] }\end{array}$ & $\begin{array}{l}\text { Study \&analysis of } \\
\text { Electrocardiogram } \\
\text { signal using } \\
\text { MATLAB \& } \\
\text { Labview as } \\
\text { effective tools }\end{array}$ & $\begin{array}{l}\text { Wavelet } \\
\text { transform } \\
\text { Digital filter } \\
\text { approach } \\
\text { Non-adaptive } \\
\text { filter } \\
\text { QRS complex } \\
\text { detection } \\
\text { Heart rate } \\
\text { detection } \\
\text { Abnormalities } \\
\text { detection } \\
\text { Calculating } \\
\text { the interval } \\
\text { and peaks of } \\
\text { each } \\
\text { waveform }\end{array}$ & $\begin{array}{l}\text { Wavelet transform } \\
\text { approach is more } \\
\text { easier and } \\
\text { convenient. } \\
\text { Non-adaptive } \\
\text { filter is also more } \\
\text { useful then } \\
\text { adaptive filter }\end{array}$ \\
\hline $\begin{array}{l}\text { Can Ye } \\
\text { et al. } \\
\text { [29] }\end{array}$ & $\begin{array}{l}\text { Classification of } \\
\text { ECG based upon } \\
\text { Heart beat using } \\
\text { Morphological and }\end{array}$ & $\begin{array}{l}\text { ICA,WT } \\
\text { technique } \\
\text { SVM } \\
\text { technique use }\end{array}$ & $\begin{array}{l}\text { Class oriented } \\
\text { accuracy-99.3\% } \\
\text { Subject oriented } \\
\text { accuracy-86.4\% }\end{array}$ \\
\hline
\end{tabular}

\begin{tabular}{|c|c|c|c|}
\hline & dynamic features & $\begin{array}{l}\text { for } \\
\text { Extract } \\
\text { features } \\
\text { related to } \\
\text { morphology } \\
\text { Extract } \\
\text { information } \\
\text { regarding RR } \\
\text { interval }\end{array}$ & \\
\hline $\begin{array}{c}\text { B. } \\
\text { Mzomenos } \\
\text { Evangelos } \\
\text { et al. } \\
\text { [31] }\end{array}$ & $\begin{array}{l}\text { A low-complexity } \\
\text { ECG feature } \\
\text { extraction } \\
\text { algorithm for } \\
\text { mobile healthcare } \\
\text { applications }\end{array}$ & $\begin{array}{l}\text { Discrete } \\
\text { wavelet } \\
\text { transform } \\
\text { technique } \\
\text { Low } \\
\text { complexity } \\
\text { algorithm } \\
\text { Feature } \\
\text { extraction } \\
\text { from QTDB } \\
\text { database,PTB } \\
\text { DB and } \\
\text { SGHCD }\end{array}$ & $\begin{array}{l}\text { Extract ECG } \\
\text { features }\end{array}$ \\
\hline $\begin{array}{l}\text { Patricia } \\
\text { Melin } \\
\text { et al. } \\
\text { [28] }\end{array}$ & $\begin{array}{l}\text { A new N.N. model } \\
\text { based on the LVQ } \\
\text { algorithm for } \\
\text { classificationof } \\
\text { arrhythmias }\end{array}$ & $\begin{array}{l}\text { MIT-BIH } \\
\text { database } \\
\text { Neural } \\
\text { network with } \\
\text { LVQ } \\
\text { algorithm }\end{array}$ & $\begin{array}{l}\text { LVQ algorithm } \\
\text { with Neural } \\
\text { Network is a } \\
\text { approach to solve } \\
\text { and classify } \\
\text { complex problems }\end{array}$ \\
\hline $\begin{array}{c}\text { Edoardo } \\
\text { Pasolli } \\
\text { et al. } \\
\text { [19] }\end{array}$ & $\begin{array}{l}\text { Method based on } \\
\text { Genetic algorithm } \\
\text { for mitigating label } \\
\text { noise in ECG } \\
\text { signal classification }\end{array}$ & $\begin{array}{l}\text { Genetic } \\
\text { optimization } \\
\text { algorithm } \\
\text { MIT-BIH } \\
\text { arrhythmia } \\
\text { database }\end{array}$ & $\begin{array}{l}\text { Genetic } \\
\text { algorithms act as a } \\
\text { filter which is } \\
\text { completely } \\
\text { independent from } \\
\text { any type of } \\
\text { classifier } \\
\text { The strategy is } \\
\text { powerful to } \\
\text { restrict the } \\
\text { Propagation of } \\
\text { mistakes } \\
\text { identified with } \\
\text { mislabeled } \\
\text { examples in the } \\
\text { sign classification } \\
\text { framework even } \\
\text { in situations } \\
\text { where their } \\
\text { quality is huge, } \\
\text { the technique } \\
\text { doesn't accept any } \\
\text { from the earlier } \\
\text { class distributions } \\
\text { thus coming about } \\
\text { especially } \\
\text { appropriate for } \\
\text { ECG signal order. }\end{array}$ \\
\hline
\end{tabular}

Published By:

Blue Eyes Intelligence Engineering \& Sciences Publication

(C) Copyright: All rights reserved. 
ECG signal Analysis and Classification Techniques

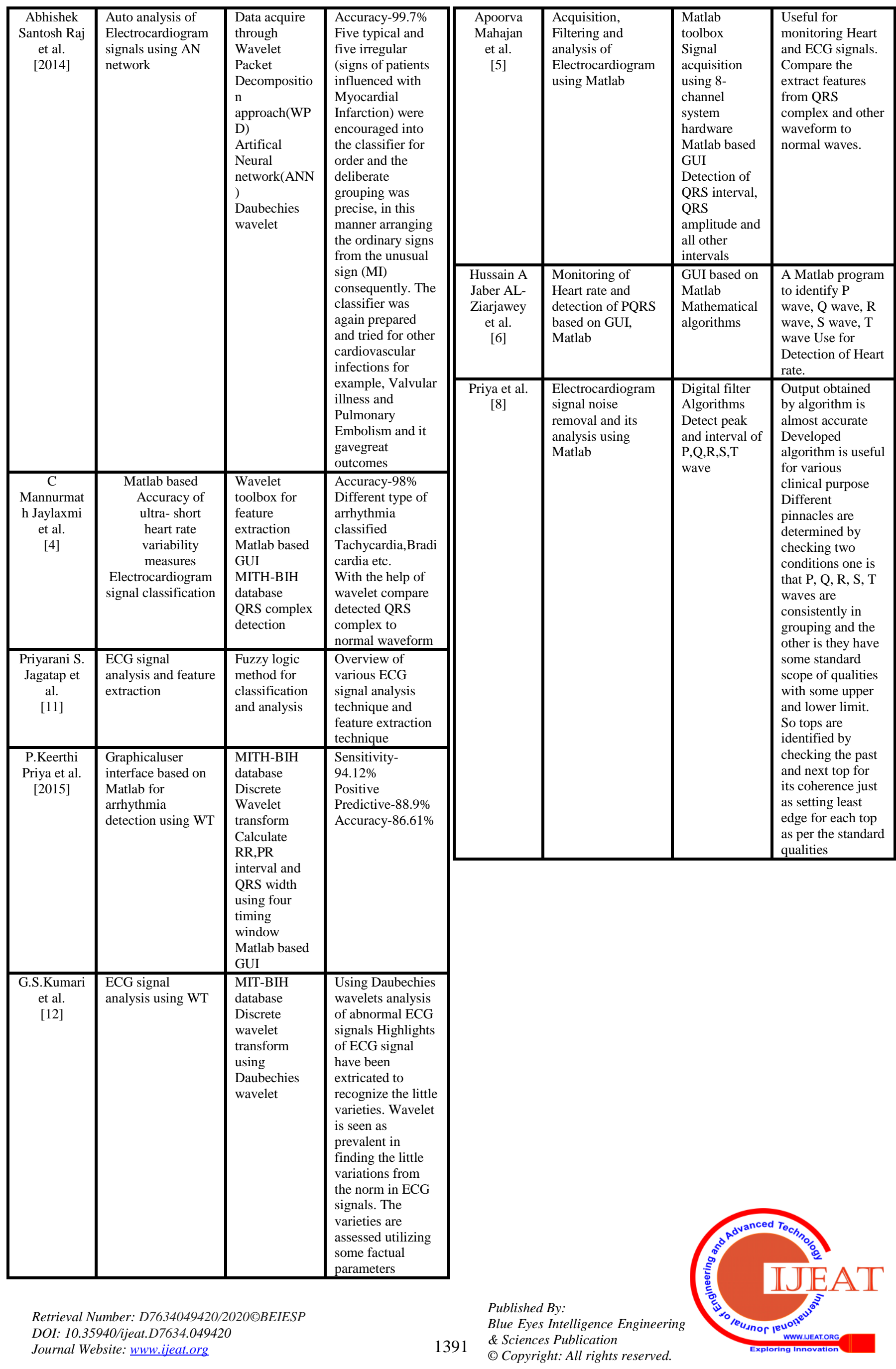




\begin{tabular}{|c|c|c|c|}
\hline $\begin{array}{l}\text { Hongqiang } \\
\text { et al. } \\
\text { [18] }\end{array}$ & $\begin{array}{l}\text { Noval } \\
\text { Electrocardiogram } \\
\text { signal classification } \\
\text { based on Kernal } \\
\text { independent } \\
\text { component analysis } \\
\text { nonlinear feature } \\
\text { extraction }\end{array}$ & $\begin{array}{l}\text { PCA and } \\
\text { KICA } \\
\text { techniques } \\
\text { used for } \\
\text { extraction of } \\
\text { features }\end{array}$ & $\begin{array}{l}\text { Up to } 720 \\
\text { gatherings of } \\
\text { datasets were } \\
\text { mimicked, also, a } \\
\text { LIBSVM tool kit } \\
\text { was utilized for } \\
\text { Characterization. } \\
\text { At last, GA was } \\
\text { embraced to } \\
\text { decide the ideal } \\
\text { parameters. The } \\
\text { last order } \\
\text { exactness is } \\
\text { 97.78\%. The } \\
\text { strategy doesn't } \\
\text { require a lot of } \\
\text { tests and is } \\
\text { superior to } \\
\text { conventional } \\
\text { strategies in } \\
\text { choosing ideal } \\
\text { highlights in the } \\
\text { multi-area space } \\
\text { vector }\end{array}$ \\
\hline $\begin{array}{l}\text { Shanshan } \\
\text { Chen } \\
\text { et al. } \\
\text { [27] }\end{array}$ & $\begin{array}{l}\text { Classification of } \\
\text { Heartbeat using } \\
\text { dynamic and } \\
\text { projected features } \\
\text { of } \\
\text { Electrocardiogram } \\
\text { signal }\end{array}$ & $\begin{array}{l}\text { DC } \\
\text { transform(Dis } \\
\text { crete cosine) } \\
\text { Support } \\
\text { vector } \\
\text { machine } \\
\text { Derive RR } \\
\text { interval }\end{array}$ & $\begin{array}{l}\text { Class based } \\
\text { accuracy-98.46\% } \\
\text { Subject based } \\
\text { accuracy-93.1\% } \\
\text { The"class-based" } \\
\text { evaluation results } \\
\text { can be seen as the } \\
\text { performance of } \\
\text { classifier prepared } \\
\text { by pulses from the } \\
\text { individuals to be } \\
\text { diagnosed. And } \\
\text { the "subject- } \\
\text { based" appraisal } \\
\text { results can be } \\
\text { deciphered as love } \\
\text { useful execution } \\
\text { of the brought } \\
\text { calculation spent } \\
\text { in a real world } \\
\text { application. }\end{array}$ \\
\hline $\begin{array}{l}\text { A. Elhaja } \\
\text { Fatin } \\
\text { et al. } \\
\text { [25] }\end{array}$ & $\begin{array}{l}\text { Arrhythmia } \\
\text { recognition and } \\
\text { classification using } \\
\text { combined linear } \\
\text { and nonlinear } \\
\text { features of } \\
\text { Electrocardiogram } \\
\text { signals }\end{array}$ & $\begin{array}{l}\text { Support } \\
\text { vector } \\
\text { machine and } \\
\text { Radial basis } \\
\text { function } \\
\text { method } \\
\text { Neural } \\
\text { network } \\
\text { QRS complex } \\
\text { detection } \\
\text { MITH-BIH } \\
\text { arrhythmia } \\
\text { database, } \\
\text { Differentiate } \\
\text { different class } \\
\text { of arrhythmia } \\
\text { like N type, S } \\
\text { type, F type, } \\
\text { V type }\end{array}$ & $\begin{array}{l}\text { Exactness - } \\
98.91 \% \\
\text { Neural network is } \\
\text { more when (PCA- } \\
\text { DWT) + (ICA- } \\
\text { HOS) features are } \\
\text { given as input. } \\
\text { NN and SVM- } \\
\text { RBF provided } \\
\text { equal affectability, } \\
\text { particularity and } \\
\text { precision (98.9\%). }\end{array}$ \\
\hline $\begin{array}{c}\text { Sandeep } \\
\text { Bhanwala } \\
\text { et al. } \\
\text { [17] }\end{array}$ & $\begin{array}{l}\text { A Study on HRV } \\
\text { Using Time and } \\
\text { Frequency Domain }\end{array}$ & $\begin{array}{l}\text { Support } \\
\text { Vector } \\
\text { Machine } \\
\text { Discrete } \\
\text { wavelet } \\
\text { transform } \\
\text { FT } \\
\end{array}$ & $\begin{array}{l}\text { Comparison of } \\
\text { different } \\
\text { techniques for } \\
\text { Heart rate } \\
\text { variability for } \\
\text { ECG signal } \\
\text { analysis }\end{array}$ \\
\hline $\begin{array}{l}\text { Joao Paulo } \\
\text { R.R. Leite } \\
\qquad[21]\end{array}$ & $\begin{array}{l}\text { Heartbeat } \\
\text { classification with } \\
\text { low computational } \\
\text { cost using Hjorth } \\
\text { parameters }\end{array}$ & $\begin{array}{l}\text { Detect RR } \\
\text { interval } \\
\text { Detect } \\
\text { amplitude } \\
\text { features } \\
\text { Detect Hjorth } \\
\end{array}$ & Accuracy-90.4\% \\
\hline
\end{tabular}

\section{CONCLUSION}

\begin{tabular}{|c|c|c|c|}
\hline & & $\begin{array}{l}\text { parameters } \\
\text { RBF-Kernal } \\
\text { SVM to } \\
\text { classify } \\
\text { heartbeat }\end{array}$ & \\
\hline $\begin{array}{l}\text { Sandeep Raj } \\
\text { et al. } \\
\text { [22] }\end{array}$ & $\begin{array}{l}\text { Electrocardiogram } \\
\text { Signal Analysis } \\
\text { Using DOST Based } \\
\text { DCT \& PSO } \\
\text { Optimized SVM }\end{array}$ & $\begin{array}{l}\text { Extract } \\
\text { features based } \\
\text { on time- } \\
\text { frequency } \\
\text { parameters } \\
\text { using Support } \\
\text { vector } \\
\text { machine } \\
\text { classifier } \\
\text { Detect RR } \\
\text { interval } \\
\text { information } \\
\text { MIT-BIH } \\
\text { arrhythmia } \\
\text { database }\end{array}$ & Accuracy-98.82\% \\
\hline $\begin{array}{l}\text { Arjun Singh } \\
\text { Vijoriya et } \\
\text { al. } \\
\quad[10]\end{array}$ & $\begin{array}{l}\text { ElectrocardiogramS } \\
\text { ignal Acquisition, } \\
\text { Extraction of } \\
\text { Features and } \\
\text { analysis of HRV } \\
\text { using } \\
\text { BIOMEDICAL } \\
\text { WORKBENCH }\end{array}$ & $\begin{array}{l}\text { Arduino Uno } \\
\text { with labview } \\
\text { is utilized for } \\
6 \text { channel } \\
\text { ADC } \\
\text { Biomedical } \\
\text { workbench id } \\
\text { used for the } \\
\text { extraction of } \\
\text { features }\end{array}$ & $\begin{array}{l}\text { Analyse the heart } \\
\text { rate variability }\end{array}$ \\
\hline $\begin{array}{l}\text { D.Raj Balaji } \\
\text { et al. } \\
\qquad[1]\end{array}$ & $\begin{array}{l}\text { Electrocardiogram } \\
\text { Signals } \\
\text { Classification using } \\
\text { SVM and Fuzzy C } \\
\text { Means Clustering }\end{array}$ & $\begin{array}{l}\text { Classification } \\
\text { techniques } \\
\text { SA-SVM \& } \\
\text { KNN } \\
\text { MIT-BIH } \\
\text { arrhythmia } \\
\text { database }\end{array}$ & $\begin{array}{l}\text { Accuracy-95\% } \\
\text { The Performance } \\
\text { Metrics like, } \\
\text { Sensitivity, } \\
\text { explicitness, } \\
\text { Accuracy, } \\
\text { Precision and } \\
\text { Recall were } \\
\text { utilized in this } \\
\text { paper to look at } \\
\text { the proposed ECG } \\
\text { order System } \\
\text { dependent on the } \\
\text { executed } \\
\text { outcomes. }\end{array}$ \\
\hline $\begin{array}{l}\text { Varun } \\
\text { Gupta et al. } \\
\text { [2] }\end{array}$ & $\begin{array}{l}\text { ECG Signal } \\
\text { Analysis: Past, } \\
\text { Present and Future }\end{array}$ & $\begin{array}{l}\text { Computer } \\
\text { aided } \\
\text { diagnosis } \\
\text { MIT-BIH } \\
\text { database }\end{array}$ & $\begin{array}{l}\text { Comparison of } \\
\text { different } \\
\text { techniques and } \\
\text { their accuracy }\end{array}$ \\
\hline
\end{tabular}

\section{COMPARISON OF RESULTS}

\begin{tabular}{|r|l|l|}
\hline \multicolumn{1}{|c|}{$\begin{array}{l}\text { Sr. } \\
\text { No }\end{array}$} & \multicolumn{1}{|c|}{ Techniques } & \multicolumn{1}{c|}{ Result } \\
\hline 1. & $\begin{array}{l}\text { Wavelet transformation technique with } \\
\text { Probabilistic Neural Network(PNN) }\end{array}$ & $\begin{array}{l}\text { Gives 99.65\% } \\
\text { accuracy in result }\end{array}$ \\
\hline 2. & $\begin{array}{l}\text { Classification techniques SA-SVM \& } \\
\text { KNN }\end{array}$ & $\begin{array}{l}\text { Gives 95\% accuracy } \\
\text { in result }\end{array}$ \\
\hline 3. & $\begin{array}{l}\text { DOST Based DCT \& PSO Optimized } \\
\text { SVM }\end{array}$ & $\begin{array}{l}\text { Gives 98.82\% } \\
\text { accuracy in result }\end{array}$ \\
\hline 4. & $\begin{array}{l}\text { Discrete cosine transform } \\
\text { Gives 98.46\% } \\
\text { accuracy in result }\end{array}$ \\
\hline 5. & PCA and KICA techniques & $\begin{array}{l}\text { Gives 97\% accuracy } \\
\text { in result }\end{array}$ \\
\hline 6. & $\begin{array}{l}\text { Wavelet Packet Decomposition } \\
\text { approach,Artificial Neural } \\
\text { network(ANN),Daubechies wavelet }\end{array}$ & $\begin{array}{l}\text { Gives 99.7\% accuracy } \\
\text { in result }\end{array}$ \\
\hline 7. & $\begin{array}{l}\text { Extreme LM classifier with SVM } \\
\text { Accuracy-89.78\% }\end{array}$ & \\
\hline
\end{tabular}

Published By:

Blue Eyes Intelligence Engineering

\& Sciences Publication

(C) Copyright: All rights reserved.

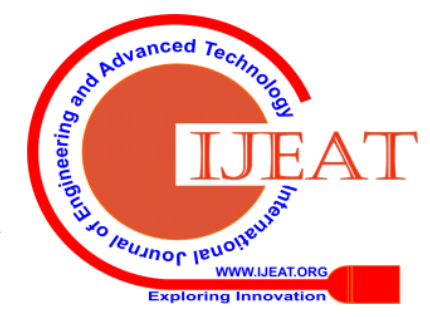


ECG signs can be examined by various methods. Grouping of ECG signal assume a significant job in observing and diagnosing cardio coherent and non-cardio sensible ailment. Both MATLAB and LabVIEW have colossal impact on ECG signal preparing. They are so valuable and helpful that indeed, even one can screen his/her heart condition just using the intensity of MATLAB and additionally LabVIEW without having an ECG machine and furthermore self determination is conceivable. All these models and systems that are talked about here can be truly valuable for trial/lab reason even we don't have any ECG information we despite everything can reproduce and examine it. In this introduced paper, present a survey of various methods for order and investigation of ECG signals.

\section{REFERENCES}

1. Balaji D. Raj, Marimuthu A. (2018), "Classification of ECG Signals Using Self Advising Support Vector Machine and Fuzzy C Means Clustering",International Journal of Research in Advent Technology.

2. Varun Gupta,Monika Mittal,ECG Signal Analysis: Past, Present and Future,978-1-5386-7339-3/18/\$31.00 @2018 IEEE

3. Islam M.K. , Haque A.N.M.M et al.(2012),"Study and Analysis of ECG Signal Using MATLAB \& LABVIEW as Effective Tools",International Journal of Computer and Electrical Engineering, Vol. 4, No. 32012

4. C Mannurmath Jaylaxmi and Prof. Raveendra(2014), "MATLAB Based ECG Signal Classification"International Journal of Science, Engineering and Technology Research , Volume 3, Issue 7,2014

5. Mahajan Apoorva, Batni Ankita, Dongre Mayuri(2015),"Acquisition, Filtering and Analysis of ECG Using MATLAB",Internationa Journal of Advanced Research in Electronics and Communication Engineering, Volume 4, Issue 5,2015

6. Jaber AL-Ziarjawey A. Hussain and Çankaya Ilyas(2015),"Heart Rate Monitoring and PQRST Detection Based on Graphical User Interface with Matlab",International Journal of Information and Electronics Engineering, Vol. 5, No. 4, 2015

7. Zigel Yaniv et al. (2000),"ECG Signal Compression Using Analysis by Synthesis Coding",IEEE TRANSACTIONS ON BIOMEDICAL ENGINEERING, VOL. 47, NO. 10,2000

8. Priya et al(2015)."MATLAB Based ECG Signal Noise Removal and its Analysis",December 2015

9. Saritha C., Sukanya V.(2008),’ECG Signal Analysis Using Wavelet Transforms",February 2008

10. Vijoriya Arjun Singh and Dr. Maheshwari Ranjan(2018),"ECG SIGNAL ACQUISITION, FEATURE EXTRACTION AND HRV ANALYSIS USING BIOMEDICAL WORKBENCH",International Journal of Advanced Research in Engineering and Technology ,Volume 9, Issue 3, May - June 2018

11. Jagatap Priyarani S and Jagtap Rupali R.(2014),”Electrocardiogram (ECG) Signal Analysis and Feature Extraction: A Survey",International Journal of Computer Sciences and Engineering 2014

12. G.S.Kumari et al. (2015),"Electrocardiographic Signal Analysis Using Wavelet Transforms",International Conference on Electrical, Electronics, Signals, Communication and Optimization (EESCO)

13. Narayana K.V.L. et al,"Wavelet based QRS detection in ECG using MATLAB",www.iiste.org, 2011

14. Yang Shengkai, Shen Haibin(2013)," Institute of VLSI Design Zhejiang University Hangzhou, China,\| Heartbeat Classification using Discrete Wavelet Transform and Kernel Principal Component Analysis", IIEEE (Tencon - Spring) 2013

15. Liu C., Wechsler H.(1998),"Enhanced Fisher linear discriminan models for face recognition", In: Proceedings of International Conference on Pattern Recognition,pp.1368-1372,1998

16. J. Tompkins W. (1993), "Biomedical Digital Signal Processing",Prentice-Hall, New Jersey, 1993

17. Sandeep Bhanwala et al.,"A Study on Heart Rate Variability Using Time and Frequency Domain",International Journal of Scientific Research in Science and Technology, 2016

18. Hongqiang L et al, "Novel ECG Signal Classification Based on KICA Nonlinear Feature Extraction", 2014

19. Edoardo Pasolli et al ,"Genetic algorithm-based method for mitigating label noise issue in ECG signal classification",www.elsevier.com/locate/bspc 2014.
20. Ping Cheng et al, "Life-Threatening Ventricular Arrhythmia Detection with Personalized Features", IEEE Access,2017

21. João Paulo R.R. Leite et al, "Heartbeat classification with low computational cost using Hjorth parameters",IETSignal Process., 2018, Vol. 12, Iss. 4, pp. 431-438

22. Sandeep Raj et al, "ECG Signal Analysis Using DCT-Based DOST and PSO Optimized SVM",0018-9456 (C) 2017 IEEE

23. Philip de Chazal et al, "Automatic Classification of Heartbeats Using ECG Morphology and Heartbeat Interval Features", IEEE Transaction on Biomedical Engineering ,VOL.51, NO.7, 2004

24. Mariano Llamedo,"Heartbeat Classification Using Feature Selection Driven by Database Generalization Criteria", IEEE Transaction on Biomedical Engineering,VOL. 58, NO. 3, MARCH 2011

25. A Fatin. Elhaja et al. (2016),"Arrhythmia recognition and classification using combined linear and nonlinear features of ECG signals".

26. Ivaylo Christov et al.(2006),"Comparative study of morphological and time-frequency ECG descriptors for heartbeat classification", (C) 2006 IPEM

27. Shanshan Chen et al. (2016),"Heartbeat classification using projected and dynamic features of ECG signal",www.elsevier.com/locate/bspc 2016

28. Patricia Melin et al.(2014),"A new neural network model based on the LVQ algorithm 4 for multi-class classification of arrhythmias".

29. Can Ye et al.(2012),"Heartbeat Classification Using Morphological and Dynamic Features of ECG Signals", IEEE TRANSACTIONS ON BIOMEDICAL ENGINEERING, VOL.59, NO. 10, OCTOBER 2012

30. Yu Sung-Nien, Chen Ying-Hsiang (2007),"Electrocardiogram beat classification based on wavelet transformation and probabilistic neural network ".

31. Arthanari M. , Karpagachelvi S., and Sivakumar M. (2011)," Classification of electrocardiogram signals with support vector machines and extreme learning machine".

32. B. Mazomenos Evangelos, Biswas Dwaipayan, Acharyya Amit et al. (2013),"A Low-Complexity ECG Feature Extraction Algorithm for Mobile Healthcare Applications "IEEE JOURNAL OF BIOMEDICAL AND HEALTH INFORMATICS, VOL. 17, NO. 2, MARCH 2013

33. R. G. Stockwell, L. Mansinha, and R. P. Lowe, "Localization of thecomplex spectrum: The S transform," IEEE Trans. Signal Process.,vol. 44, no. 4, pp. 998-1001, Apr. 1996.

34. R. G. Stockwell, "A basis for efficient representation of the Stransform," Digit. Signal Process., vol. 17, no. 1, pp. 371-393, May 2006.

35. Atal, D.K., Singh, M. A hybrid feature extraction and machine learning approaches for epileptic seizure detection. Multidim Syst Sign Process 31, 503-525 (2020)

36. https://doi.org/10.1007/s11045-019-00673-4.

37. Atal, D.K., Singh, M. A dictionary matrix generation based compression and bitwise embedding mechanisms for ECG signal classification. Multimed Tools Appl (2020).

38. https://doi.org/10.1007/s11042-020-08671-6.

\section{AUTHOR(S) PROFILE:}

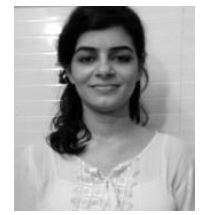

Seema Punia received her B.Tech. Degree in Biomedical Engineering in 2018 from Deenbandhu Chhotu Ram University of Science and Technology (DCRUST) Murthal Sonipat, Haryana India and presently Pursuing M.Tech. Degree in Biomedical Engineering from DCRUST Murthal Sonipat, Haryana India. Seema research's interests include physiological analysis, processing, feature extraction and classification tasks of biomedical signals.

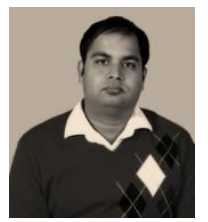

Dinesh Kumar Atal received B.Tech. Degree in Biomedical Engineering in 2005 from Guru Jambheshwar University of Science and Technology, Hisar (Haryana) India and M.Tech. Degree in Electrical Engineering with specialization in Instrumentation and Control from Deenbandhu Chhotu Ram University of Science and Technology, Sonipat (Haryana) India in

2011.

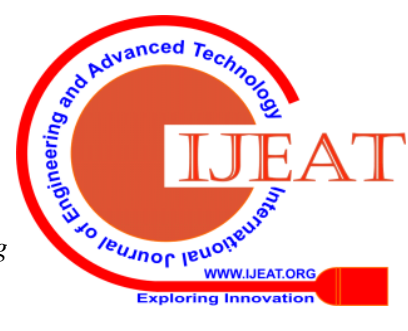


Dinesh's research interests include machine learning, data compression, filtering, classification algorithms, signals and image processing of human physiological signals. He has research publications in several journals and conferences on ECG and EEG signals analysis and processing.

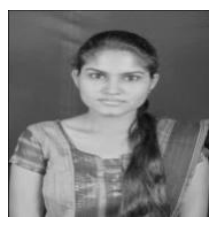

Sarita Singh received B.Tech. Degree in Biotechnology in 2010 from U.I.E.T. Maharishi Dayanand University Rohtak (Haryana) India and M.Tech. Degree in Biomedical Engineering from Motilal Nehru National institute of Technology Allahabad (U.P.) in 2012. Presently, Sarita is pursuing Ph.D. in Biomedical Engineering from Motilal Nehru National institute of Technology Allahabad (U.P.). Her research interests include biomaterials, biomechanics, Comsol multi-physics. She has published several journal and conference paper in biomaterials, biomechanics and modeling simulation by Comsol multi-physics. Sarita received best poster award in National conference at NIT Kurukshetra in 2013.

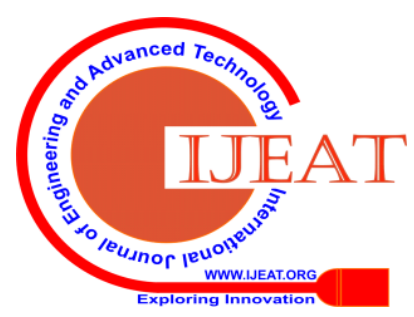

